\title{
Association of Periprocedural Phentolamine Infusion with Favorable Outcome in Patients with Chronic Kidney Disease and Chronic Coronary Syndrome undergoing coronary catheterization: A Prospective Randomized Controlled Study
}

Mohamed Abo Hamila ( $\square$ hamila@hotmail.com )

Beni-Suef University

Helmy El Ghawaby

Cairo University hospitals

Mohamed Zaki

National Heart Institute

Mohamed Soliman

Cairo University hospitals

Khaled Gabr

National Heart Institute

Research Article

Keywords: Contrast Induced Nephropathy, Phentolamine, Coronary Catheterization

Posted Date: March 22nd, 2022

DOI: https://doi.org/10.21203/rs.3.rs-1329767/v2

License: (c) (1) This work is licensed under a Creative Commons Attribution 4.0 International License.

Read Full License 


\section{Abstract}

\section{Background}

Chronic kidney disease (CKD) is a major risk factor for contrast induced nephropathy acute kidney injury (CIN-AKI) in chronic coronary syndrome (CCS) patients undergoing coronary catheterization. We aimed to evaluate the efficacy of phentolamine in prevention of CIN-AKI in CKD and CCS patients undergoing percutaneous coronary catheterization for diagnostic angiography \pm stenting.

\section{Methods}

Participants with CKD and CCS planned for percutaneous coronary catheterization were included, while participants with normal kidney functions were excluded. A consecutive sample of 107 participants (mean age $58.62 \pm 8.96$ years, $64.5 \%$ males) was selected, underwent diagnostic coronary angiography or percutaneous coronary intervention, and received either conventional CIN-AKI prevention strategy (group 1) or periprocedural phentolamine and conventional CIN-AKI prevention strategy (group 2).

\section{Results}

The percentages of study participants who had CIN-AKI were $82.9 \%$ for group 1 and $17.1 \%$ for group 2 , respectively. There were a statistically significant association between periprocedural phentolamine and prevention of CIN-AKI $(\mathrm{OR}=0.041,95 \% \mathrm{Cl} 0.0149-0.1128, \mathrm{P}<0.0001)$ and statistically significant differences between group 1 versus group 2 regarding the urine output $(\mathrm{ml} / \mathrm{kg})$ and the urine output ( $\mathrm{ml} /$ hour) within 72 hours post procedure, the peak change in serum creatinine within 72 hours post procedure, the percentage of change relative to the baseline serum creatinine at 72 hours post procedure, and the incidence rate of major adverse cardiac and cerebrovascular events within 90 days post procedure $(\mathrm{t}(105)=-0.69, \mathrm{p}<0.001, \mathrm{t}(105)=-52.46, \mathrm{p}<0.001, \mathrm{t}(102)=0.2, \mathrm{p} \mathrm{0.018,t} \mathrm{t}(102)=23.54, \mathrm{p}<0.001$, and $t(102)=1.168, P<0.001$, respectively).

\section{Conclusions}

The incidence of CIN-AKI and the peak change in serum creatinine within 72 hours post procedure was lower in CKD patients who underwent coronary catherization and received phentolamine infusion versus intravenous saline. The significant association of phentolamine infusion with prevention of CIN-AKI highlights the potential role of phentolamine for protection of the kidney in CKD patients planned for coronary catherization.

Trial Registration Number:

$1-111015$

Date of Trial Registration: 01/06/2016 


\section{Introduction}

Contrast induced nephropathy (CIN) is a leading cause of hospital-acquired acute kidney injury (AKI) with a reported incidence rate of $1 \%-6 \%$ in the general population [1]. More than half of the contrast induced nephropathy acute kidney injury (CIN-AKI) cases are reported in patients undergoing percutaneous coronary catheterization for diagnostic angiography \pm stenting. A large, retrospective case-control study at Mayo clinic showed an overall 3.3\% incidence rate of CIN-AKI in patients who underwent primary percutaneous coronary intervention (PCI) [2]. Risk factors for CIN-AKI include chronic kidney disease (CKD), diabetes mellitus (DM), age, hypertension, peripheral vascular disease (PVD), congestive heart failure (CHF), shock with systolic blood pressure $<100 \mathrm{mmHg}$, intra-aortic balloon pump, anemia with hemoglobin $<11 \mathrm{mg} / \mathrm{dl}$, serum creatinine $>1.5 \mathrm{mg} / \mathrm{dl}$, and contrast media (CM) volume $>260 \mathrm{ml}[3,4]$. In 1992 , Hall et al showed that CIN-AKI is 30 times higher in patients with baseline serum creatinine $\geq 2.0$ $\mathrm{mg} / \mathrm{dl}$ versus patients with baseline serum creatinine $\leq 1.2 \mathrm{mg} / \mathrm{dl}$ [5]. CIN-AKI in CKD patients undergoing percutaneous coronary catheterization for diagnostic angiography \pm stenting is associated with an overall mortality rate of $7 \%-31 \%$, dialysis treatment rate of less than $1 \%$, and permanent dialysis treatment rate of less than $0.13 \%$ [6]. We wanted to evaluate the efficacy of phentolamine in prevention of CIN-AKI in patients with CKD and chronic coronary syndrome (CCS) undergoing diagnostic coronary angiography or $\mathrm{PCl}$.

\section{Methods}

\section{Study Design:}

Our study was a 3-year prospective, open-labeled, randomized, controlled study conducted at a single critical care unit (CCU) in a tertiary care hospital. This study was performed in accordance with the Egyptian National Commission for Bioethics (National Commission for UNESCO) statement on ethical conduct in human research, study procedures were carried out following the Code of Ethics of the World Medical Association (Declaration of Helsinki), study design and protocol were reviewed and approved by the human ethics committee of Cairo university, study was registered and issued a trial registration number (I-111015), study participants signed written informed consents, study data was anonymized, and the privacy rights of the study participants were observed diligently.

\section{Study Participants:}

Study participants were CKD and CCS patients, candidate for diagnostic coronary angiography or $\mathrm{PCl}$, and referred to the CCU. The study participants were subjected to history taking and data collection for age, gender, hypertension, DM, dyslipidemia, smoking, cerebrovascular disease, PVD, acute coronary syndrome (ACS), prior coronary angiography, and prior revascularization procedure. In addition, study participants were subjected to comprehensive clinical examination including measurement of vital signs on admission (systolic and diastolic blood pressure, heart rate, respiratory rate, and body temperature), mean arterial blood pressure, body weight and height, admission and discharge twelve-lead electrocardiograms (ECGs), transthoracic echocardiography (TTE), complete blood count, fasting blood 
glucose, blood urea, serum creatinine, total cholesterol, high density lipoprotein cholesterol, low density lipoprotein cholesterol, triglycerides, creatinine kinase (CK), creatine kinase-MB (CK-MB), and troponin. Normal values for ECG waves and intervals were referenced to the American College of Cardiology/American Heart Association Task Force on Assessment of Diagnostic and Therapeutic Cardiovascular Procedures (Committee on Electrocardiography) report [7]. Data documented with ECGs included arrhythmias, new bundle branch block, ST segment elevation, ST segment depression, changes of T wave, or no significant changes. Data documented with TTE included left ventricular ejection fraction (LVEF). The preprocedural CKD was categorized based on the estimated glomerular filtration rate (eGFR) using the simplified Modification of Diet in Renal Disease (MDRD) formula into mild CKD (60-89 $\left.\mathrm{ml} / \mathrm{min} / 1.73 \mathrm{~m}^{2}\right)$, moderate CKD $\left(30-59 \mathrm{ml} / \mathrm{min} / 1.73 \mathrm{~m}^{2}\right)$, and severe CKD $\left(15-29 \mathrm{ml} / \mathrm{min} / 1.73 \mathrm{~m}^{2}\right)$, respectively [8]. Screened participants were enrolled if they had CKD, CCS after previous episode of ACS including ST segment elevation myocardial infarction (STEMI), non-ST segment elevation myocardial infarction (NSTEMI), or unstable angina (UA), and planned for diagnostic coronary angiography or $\mathrm{PCl}$. Screened participants with normal kidney functions, single functioning kidney, end stage renal disease (ESRD) on regular dialysis, history of kidney transplant, AKI triggered by cocaine, surgery, sepsis, trauma, or cardiogenic shock, pulmonary edema, acute heart failure with LVEF $<30 \%$, systolic blood pressure $<80$ $\mathrm{mmHg}$, bronchial asthma, multiple myeloma, pregnancy, allergy to phentolamine or $\mathrm{CM}$, or received barbiturates, antipsychotic agents, phosphodiestrase- 5 inhibitors, CM within 7 days of study entry, or aadrenoreceptor blocker at the time of admission were excluded from the study.

\section{Study Procedures:}

One hundred and seven eligible participants were randomly, consecutively assigned with an equal 1:1 allocation ratio into an open-labeled unblinded fashion. The enrolled study participants were randomized into 2 groups of 52 participants for conventional CIN-AKI prevention strategy with normal saline infusion at a rate of $1-1.5 \mathrm{ml} / \mathrm{kg} / \mathrm{hr}$ to be started 12 hours before coronary angiography or $\mathrm{PCl}$ and continued for 24 hours after $\mathrm{CM}$ exposure in addition to 2 doses of $\mathrm{N}$-acetylcysteine $600-1200 \mathrm{mg}$ per oral the day before admission and 2 doses on the day of the procedure (Group 1) versus 55 age and gender matched participants for phentolamine 1-5 mg intravenous (IV) bolus followed by continuous IV infusion at a rate of $0.5-20 \mu \mathrm{gm} / \mathrm{kg} / \mathrm{min}$ to be started 1 hour before coronary angiography or $\mathrm{PCl}$ and continued for 4-6 hours after $\mathrm{CM}$ exposure in addition to $\beta$-adrenoceptor blocker and conventional CIN-AKI prevention strategy (Group 2).

\section{End Points:}

The study evaluated the incidence of $\mathrm{CIN}-\mathrm{AKI}$, association of periprocedural phentolamine with prevention of CIN-AKI, change in urine output, serum creatinine and eGFR, and incidence of major adverse cardiac and cerebrovascular events (MACCE) in CKD patients undergoing coronary catheterization.

\section{Statistical Analysis:}

The assessment outcomes were coded, and the data was analyzed with the Statistical Package for the Social Sciences software (SPSS ${ }^{\circledR}$ ) version 25. Quantitative (continuous) data was expressed as means 
and standard deviations, while qualitative (categorial) data was expressed as frequencies and percentages. Comparisons between parametrically distributed quantitative variables were done with the Independent two-tailed t-test, between non-parametrically distributed quantitative variables with MannWhitney test, and between qualitative variables with Chi-square test, respectively $[9,10]$. The confidence interval was set to $95 \%$ and the margin of error accepted was set to $5 \%$. Any comparison considered statistically significant was at $\mathrm{P}<0.05$ or less and highly significant at $\mathrm{P}<0.01$.

\section{Results}

\section{Study Participants and Procedures:}

We recruited 107 patients from one hospital in one country from October 2016 through November 2019. The 2 study groups were balanced with regards to the baseline characteristics and risk factors (Table 1). The key sociodemographic feature of the enrolled participants was male predominance $(67.3 \%$ of group 1 and $61.8 \%$ of group 2 were males). Age was not significantly different between both groups (mean age was $58.5 \pm 7.83$ years for group 1 versus $58.73 \pm 10.03$ years for group $2, P>0.914$ ). Risk factors as hypertension, DM, smoking, ACS, prior coronary angiography, prior coronary artery bypass grafting, prior $\mathrm{PCl}$, and history of $\mathrm{CHF}$ (variables believed to cause confounding) were equally distributed (matched) among both studied groups to adjust for confounding [11]. All enrolled participants completed the study and there were no withdrawals. 
Table 1

Patients characteristics and demographic data

\begin{tabular}{|c|c|c|c|c|c|}
\hline & Gro & $1(N=52)$ & Grol & $(\mathrm{N}=55)$ & $P$ value \\
\hline & Mea & SD & Mea & & \\
\hline Age (Years) & 58.5 & 7.83 & 58.7 & 10.03 & 0.914 \\
\hline Baseline Serum Creatinine & 1.65 & 0.58 & 1.82 & & 0.847 \\
\hline Baseline eGFR & 44.5 & 10.06 & 38.4 & 9.81 & 0.002 \\
\hline Ejection Fraction & 54.7 & 11.64 & 51.6 & 10.46 & 0.233 \\
\hline & Gro & $1(N=52)$ & Grol & $(\mathrm{N}=55)$ & $P$ value \\
\hline & $\mathrm{N}$ & $\%$ & $\mathrm{~N}$ & $\%$ & \\
\hline Male & 17 & $32.7 \%$ & 21 & $38.2 \%$ & 0.553 \\
\hline Female & 35 & $67.3 \%$ & 34 & $61.8 \%$ & \\
\hline Mild CKD & 31 & $59.6 \%$ & 15 & $27.3 \%$ & 0.003 \\
\hline Moderate CKD & 17 & $32.7 \%$ & 30 & $54.5 \%$ & \\
\hline Severe CKD & 4 & $7.7 \%$ & 10 & $18.2 \%$ & \\
\hline Hypertension & 42 & $80.8 \%$ & 47 & $85.5 \%$ & 0.517 \\
\hline Smoking & 33 & $63.5 \%$ & 31 & $56.4 \%$ & 0.454 \\
\hline CABG History & 2 & $3.8 \%$ & 4 & $7.3 \%$ & 0.679 \\
\hline $\mathrm{CHF}$ & 31 & $59.6 \%$ & 28 & $50.9 \%$ & 0.365 \\
\hline STEMI & 34 & $65.4 \%$ & 44 & $80.0 \%$ & 0.250 \\
\hline NSTEMI & 12 & $23.1 \%$ & 7 & $12.7 \%$ & \\
\hline UA & 6 & $11.5 \%$ & 4 & $7.3 \%$ & \\
\hline Old PCl once & 12 & $23.1 \%$ & 11 & $20.0 \%$ & 0.703 \\
\hline Old PCI Twice & 1 & $1.9 \%$ & 3 & $5.5 \%$ & \\
\hline Old CA once & 24 & $46.2 \% \%$ & 27 & $49.1 \%$ & 0.733 \\
\hline Old CA Twice & 0 & $0.0 \%$ & 1 & $1.8 \%$ & \\
\hline CHF: Congestive Heart Failu & Coror & Angiogra & ABG: & onary Art & pass Gra \\
\hline PCl: Percutaneous coronary & ntion, & TEMI: Nor & egm & nyocardia & rction, \\
\hline STEMI: ST segment elevati & 10 & arction U & 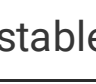 & & \\
\hline
\end{tabular}




\section{Incidence of Contrast Induced Nephropathy Acute Kidney Injury}

As per Risk, Injury, Failure, Loss, and End-stage Kidney (RIFLE) criteria, the incidence of CIN-AKI in group 1 was $82.9 \%$ versus $17.1 \%$ in group 2 . There was a significant difference in the incidence rate of CIN-AKI between both groups $(P<0.001)$.

\section{Odds of Contrast Induced Nephropathy Acute Kidney Injury}

The odds of developing CIN-AKI were $>95 \%$ lower among study participants who received periprocedural phentolamine and normal saline infusion than controls who received normal saline infusion only $(\mathrm{OR}=$ $0.041,95 \% \mathrm{Cl} 0.0149-0.1128, \mathrm{P}<0.0001)$.

\section{Change in Urine Output}

There were statistically significant differences between group 1 versus group 2 regarding the urine output $(\mathrm{ml} / \mathrm{kg})$ and urine output $(\mathrm{ml} /$ hour) within 72 hours post procedure $(\mathrm{t}(105)=-0.69, \mathrm{p}<0.001$ and $\mathrm{t}(105)=$ $-52.46, p<0.001$, respectively) (Fig. 1).

\section{Change in Serum Creatinine and estimated Glomerular Filtration Rate}

There were statistically significant differences between group 1 versus group 2 regarding the peak change in serum creatinine within 72 hours post procedure, percentage of change relative to the baseline serum creatinine at 72 hours post procedure, percentage of change relative to the baseline eGFR at 72 hours post procedure, percentage of change relative to the baseline serum creatinine at 30 days post procedure, percentage of change relative to the baseline eGFR at 30 days post procedure, percentage of change relative to the baseline serum creatinine at 90 days post procedure, and percentage of change relative to the baseline eGFR at 90 days post procedure $(t(102)=0.2, p 0.018, t(102)=23.54, p<0.001$, $t(102)=-19.34, p<0.001, t(102)=16.53, p<0.001, t(102)=-16.51, p<0.001, t(102)=18.13, p<0.001$, and $t(102)=-15.71, p<0.001$, respectively) (Figs. 2 and 3 ).

\section{Incidence of Major Adverse Cardiac and Cerebrovascular Events}

The incidence of MACCE within 90 days post procedure in group 1 was $2.75 \pm 0.926$ versus $1.582 \pm 0.994$ in group 2. There was a significant difference in the incidence rate of MACCE between both groups $(\mathrm{t}(102)=1.168, \mathrm{P}<0.001)$.

\section{Discussion}

Percutaneous coronary catheterization for angiography and stenting has been extensively performed in coronary artery disease for revascularization. CIN is a leading cause of AKI with a reported annual incidence rate of $1 \%-6 \%$ in the general population [1]. CIN-AKI is associated with significant morbidity 
and mortality and can worsen cardiac, cerebrovascular, and patient reported outcomes. CKD, DM, age, and hypertension have been suggested as risk factors for CIN-AKI [3, 4]. Periprocedural hydration with IV saline infusion is widely accepted as a cost effective CIN-AKI prevention strategy. Using a vasodilator agent for prevention of CIN-AKI was investigated by Takahide Nawa et al. in 2015 who showed a significant lower incidence of CIN-AKI in the study participants who received periprocedural hydration and nicorandil (2.0\%) versus the study participants who received periprocedural hydration only $(10.7 \%)(\mathrm{P}<$ 0.02 ) and a statistically significant lower odds of developing CIN-AKI with periprocedural hydration and nicorandil versus periprocedural hydration only (OR: $0.173,95 \% \mathrm{Cl} 0.037-0.812, \mathrm{P}=0.026)$ [12]. A recent systematic review and Bayesian network meta-analysis published in 2017 reported a statistically significant lower odds of developing CIN-AKI with either periprocedural hydration, high-dose statin, and Nacetylcysteine versus periprocedural hydration only $(\mathrm{OR}=0.31,95 \% \mathrm{Cl} 0.14-0.60)$ or periprocedural hydration and high-dose statin versus periprocedural hydration only $(\mathrm{OR}=0.37,95 \% \mathrm{Cl} 0.19-0.64)$ in sensitivity analyses, meta-regression, and subgroup analyses, respectively [13]. In 2018, Weisbord et al. investigated the efficacy of IV saline, IV sodium bicarbonate, oral N-acetylcysteine, and oral placebo. CINAKI occurred with comparable frequencies in all groups and the study concluded no differential benefit of IV saline, IV sodium bicarbonate, oral $\mathrm{N}$-acetylcysteine, or oral placebo for the prevention of death, need for dialysis, persistent kidney impairment at 90 days post procedure, prevention of CIN-AKI, or other secondary end points [14]. Another study by Xing $\mathrm{K}$ et al. showed that, despite the significant difference in the incidence rate of $\mathrm{CIN}-\mathrm{AKI}$ in the study participants who received periprocedural recombinant human Btype natriuretic peptide versus the study participants who received nitroglycerin (12.28\% versus $28.81 \%, P$ $<0.05)$, there were non-significant differences between both groups in mortality and re-hospitalization within 3 months after $\mathrm{PCl}[15]$.

Our prospective study revealed a statistically significant difference in the incidence rate of CIN-AKI as per the RIFLE criteria between the participants who received periprocedural hydration and phentolamine (17.1\%) versus the participants who received periprocedural hydration only $(82.9 \%)(P<0.001)$, a statistically significant lower odds of developing CIN-AKI with periprocedural hydration and phentolamine versus periprocedural hydration only $(\mathrm{OR}=0.041,95 \% \mathrm{Cl} 0.0149-0.1128, \mathrm{P}<0.0001)$, a statistically significant association between periprocedural phentolamine and prevention of CIN-AKI, and a statistically significant difference in the incidence rate of MACCE between both groups within 90 days post procedure $(\mathrm{t}(102)=1.168, \mathrm{P}<0.001)$.

\section{Strengths and limitations}

Our study didn't have missing data allowing robust per protocol analysis and the investigators who analyzed and reported the anonymous urine output, serum creatinine, and eGFR were blinded to the identity and clinical data of the study participants and hence minimizing observer bias. On the other hand, the study has limitations. It was a single centered study with a small sample size. Being a short prospective study with a lack of lengthy follow up didn't allow us to investigate the chronological relationship between periprocedural phentolamine infusion and the long-term all-cause morbidity and mortality following cardiac catheterization. 


\section{Conclusions And Recommendations}

CKD is a major risk factor for CIN-AKI in CCS patients undergoing coronary catheterization. The significantly lower odds of developing CIN-AKI among study participants who received periprocedural phentolamine highlights the potential role of periprocedural phentolamine infusion in improving postprocedural urine output and prevention of CIN-AKI in CKD patients. Periprocedural phentolamine resulted in lower incidence of MACCE within 90 days post procedure and reduced short-term all-cause morbidity following cardiac catheterization. Large prospective studies with lengthy follow-up are warranted to assess the chronological relationship between periprocedural phentolamine infusion and long-term all-cause morbidity and mortality following cardiac catheterization.

\section{Declarations}

\section{Ethical approval and consent to participate}

This study was reviewed and approved by the human ethics committee of Cairo university (I-111015) and informed consents were obtained from all human research subjects for participation in the study.

\section{Consent for publication}

Informed consents were obtained from all study participants for publication of all identifiable details, which can include photograph(s) and/or videos and/or case history and/or details within the text ("Material") to be published in the above Journal and Article.

\section{Availability of supporting data}

All data generated during this study are included in this published article and all datasets used and/or analysed are available from the corresponding author on reasonable request.

\section{Disclosures and competing interests}

The authors have no conflicts and/or competing interests to disclose.

\section{Funding sources}

This research did not receive any specific grant from funding agencies in the public, commercial, or notfor-profit sectors.

\section{Authors' contributions}

Mohamed Soliman designed the study, Khaled Gabr collected, analyzed and interpreted data, Mohamed Abo Hamila and Mohamed Zaki have drafted and/or critically reviewed the manuscript for important intellectual content, agreed with the revised content, and approved the submitted manuscript, Helmy El Ghawaby prepared the figures, and Mohamed Abo Hamila is responsible for the overall content as guarantor. 


\section{Acknowledgements}

Not Applicable.

\section{References}

1. Parfrey P. The clinical epidemiology of contrast-induced nephropathy. Cardiovasc Intervent Radiol. 2005;28 Suppl 2:S3-S11. doi:10.1007/s00270-005-0196-8

2. Rihal CS, Textor SC, Grill DE, et al. Incidence and prognostic importance of acute renal failure after percutaneous coronary intervention. Circulation. 2002;105(19):2259-2264.

doi:10.1161/01.cir.0000016043.87291.33

3. Chong E, Poh KK, Liang S, Tan HC. Risk factors and clinical outcomes for contrast-induced nephropathy after percutaneous coronary intervention in patients with normal serum creatinine. Ann Acad Med Singap. 2010;39(5):374-380.

4. Bartholomew BA, Harjai KJ, Dukkipati S, et al. Impact of nephropathy after percutaneous coronary intervention and a method for risk stratification. Am J Cardiol. 2004;93(12):1515-1519. doi:10.1016/j.amjcard.2004.03.008

5. Hall KA, Wong RW, Hunter GC, et al. Contrast-induced nephrotoxicity: the effects of vasodilator therapy. J Surg Res. 1992;53(4):317-320. doi:10.1016/0022-4804(92): 90054-4

6. Feldkamp T, Kribben A. Contrast media induced nephropathy: definition, incidence, outcome, pathophysiology, risk factors and prevention. Minerva Med. 2008;99(2):177-196.

7. Schlant RC, Adolph RJ, DiMarco JP, Dreifus LS, Dunn MI, Fisch C, Garson A Jr, Haywood LJ, Levine HJ, Murray JA, et al. Guidelines for electrocardiography. A report of the American College of Cardiology/American Heart Association Task Force on Assessment of Diagnostic and Therapeutic Cardiovascular Procedures (Committee on Electrocardiography). Circulation. 1992 Mar;85(3):1221-8. doi: 10.1161/01.cir.85.3.1221. PMID: 1537123.

8. Ronco C, McCullough P, Anker SD, et al. Cardio-renal syndromes: report from the consensus conference of the acute dialysis quality initiative. Eur Heart J. 2010;31(6):703-711. doi:10.1093/eurheartj/ehp507

9. Chan YH. (2003): Biostatistics 102: Quantitative Data - Parametric \& Non-parametric Tests. Singapore Med J.; 44(8): 391-396.

10. Chan YH. (2003): Biostatistics 103: Qualitative Data -Tests of Independence. Singapore Med J.; 44(10): 498-503.

11. Jager KJ, Zoccali C, Macleod A, et al. Confounding: what it is and how to deal with it. Kidney Int. 2008; 73: 256-260.

12. Nawa T, Nishigaki K, Kinomura Y, et al. Continuous intravenous infusion of nicorandil for 4 hours before and 24 hours after percutaneous coronary intervention protects against contrast-induced nephropathy in patients with poor renal function. Int J Cardiol. 2015;195:228-234.

doi:10.1016/j.ijcard.2015.05.078 
13. Su X, Xie X, Liu L, et al. Comparative Effectiveness of 12 Treatment Strategies for Preventing Contrast-Induced Acute Kidney Injury: A Systematic Review and Bayesian Network Meta-analysis. Am J Kidney Dis. 2017;69(1):69-77. doi:10.1053/j.ajkd.2016.07.033

14. Weisbord SD, Gallagher M, Jneid $\mathrm{H}$, et al. Outcomes after Angiography with Sodium Bicarbonate and Acetylcysteine. N Engl J Med. 2018;378(7):603-614. doi:10.1056/NEJMoa1710933

15. Xing K, Fu X, Wang Y, et al. Effect of rhBNP on renal function in STEMI-HF patients with mild renal insufficiency undergoing primary PCl. Heart Vessels. 2016;31(4):490-498. doi:10.1007/s00380-0150642-8

\section{Figures}
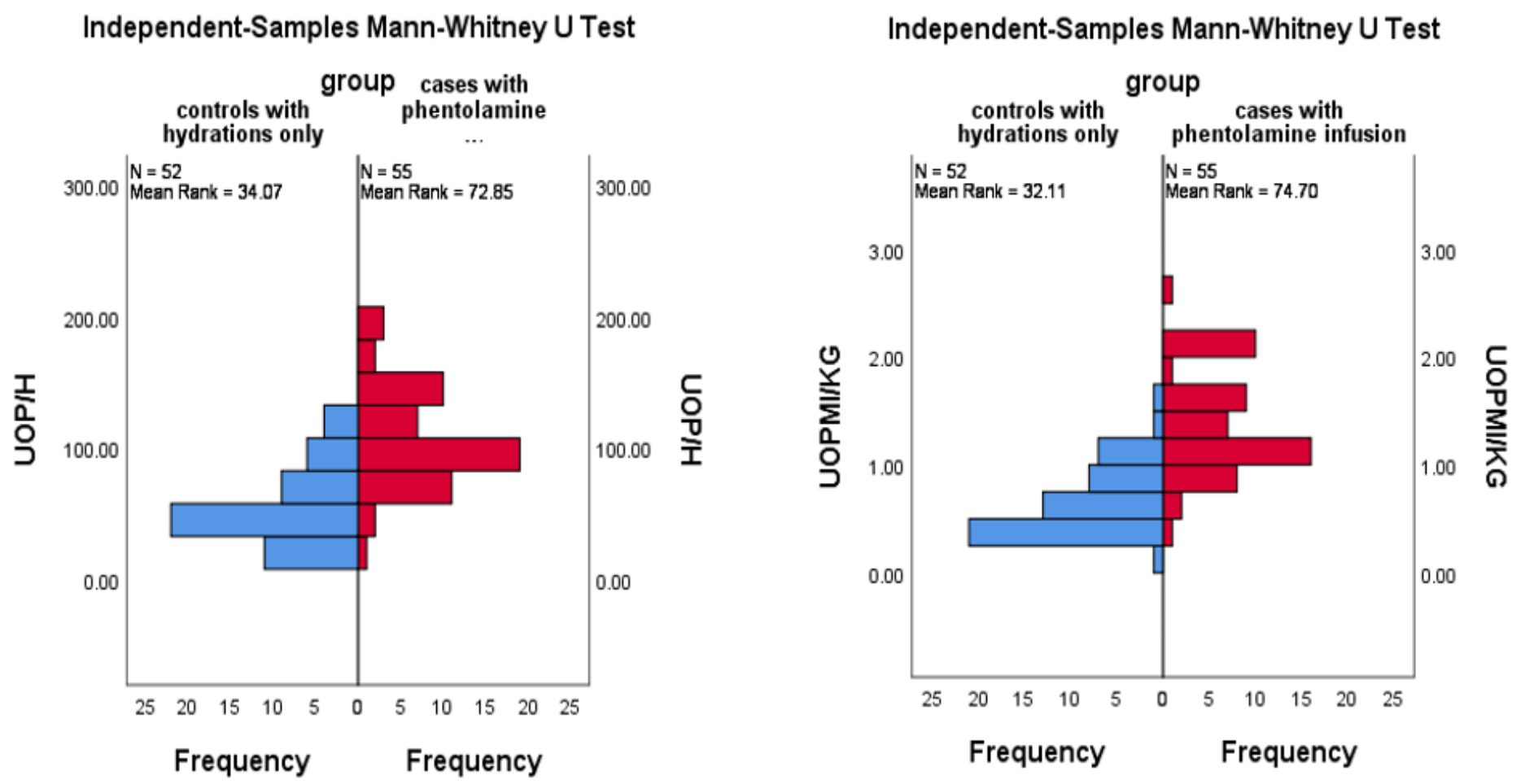

Figure 1

Change in urine output $(\mathrm{ml} / \mathrm{kg})$ and urine output $(\mathrm{ml} / \mathrm{hour})$ in group 1 versus group 2

Histogram showing the change in urine output $(\mathrm{ml} / \mathrm{kg})$ in group 1 (mean urine output $0.64 \pm 0.31 \mathrm{ml} / \mathrm{kg}$ ) versus group 2 (mean urine output $1.33 \pm 0.48 \mathrm{ml} / \mathrm{kg}$ ) and the change in urine output ( $\mathrm{ml} /$ hour) in group 1 (mean urine output $56.54 \pm 28.14 \mathrm{ml} / \mathrm{hr}$ ) versus group 2 (mean urine output $109.00 \pm 39.20 \mathrm{ml} / \mathrm{hr}$ ) within 72 hours post procedure. 


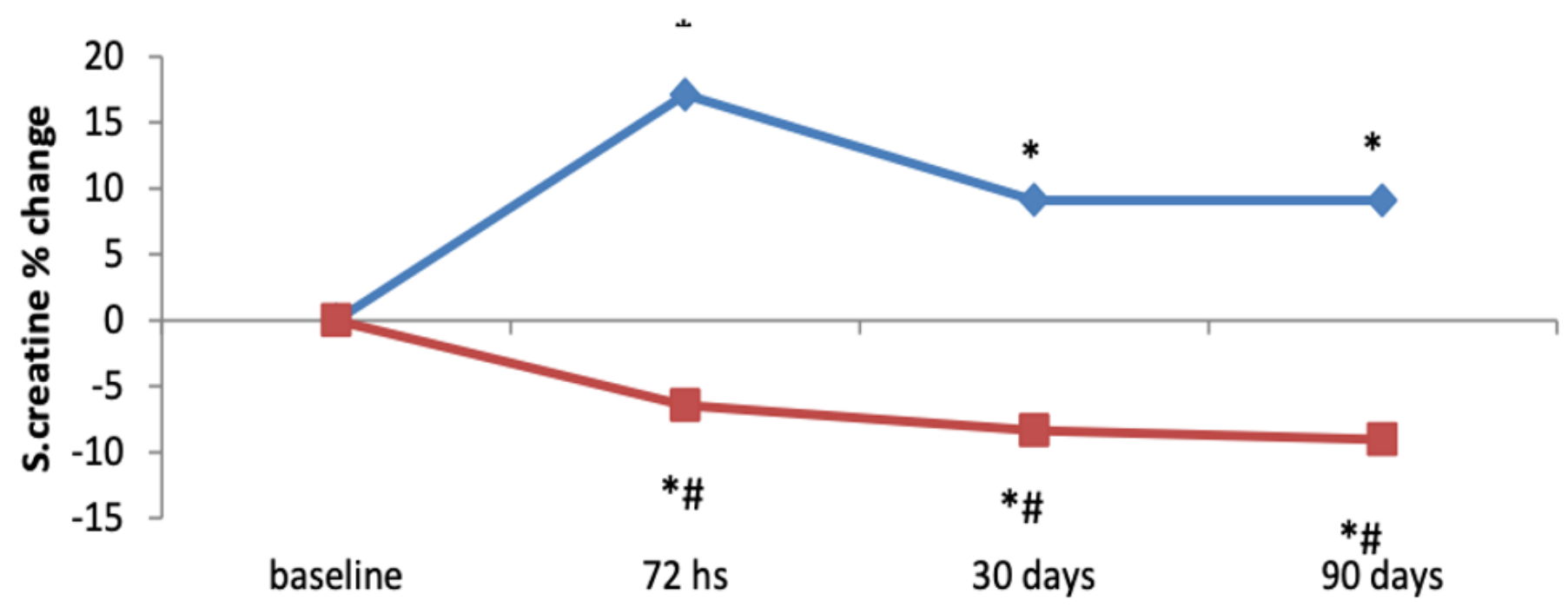

Figure 2

Change in Serum Creatinine in group 1 versus group 2

Line chart showing the percentage of change in serum creatinine relative to the baseline serum creatinine in group 1 at 72 hours post procedure (17.11 \pm 15.93$), 30$ days post procedure $(9.12 \pm 23.06)$, and 90 days post procedure $(9.10 \pm 22.22)$ versus the percentage of change in serum creatinine relative to the baseline serum creatinine in group 2 at 72 hours post procedure (-6.43 \pm 11.12$), 30$ days post procedure $(-8.34 \pm 15.83)$, and 90 days post procedure $(-9.03 \pm 15.59)$

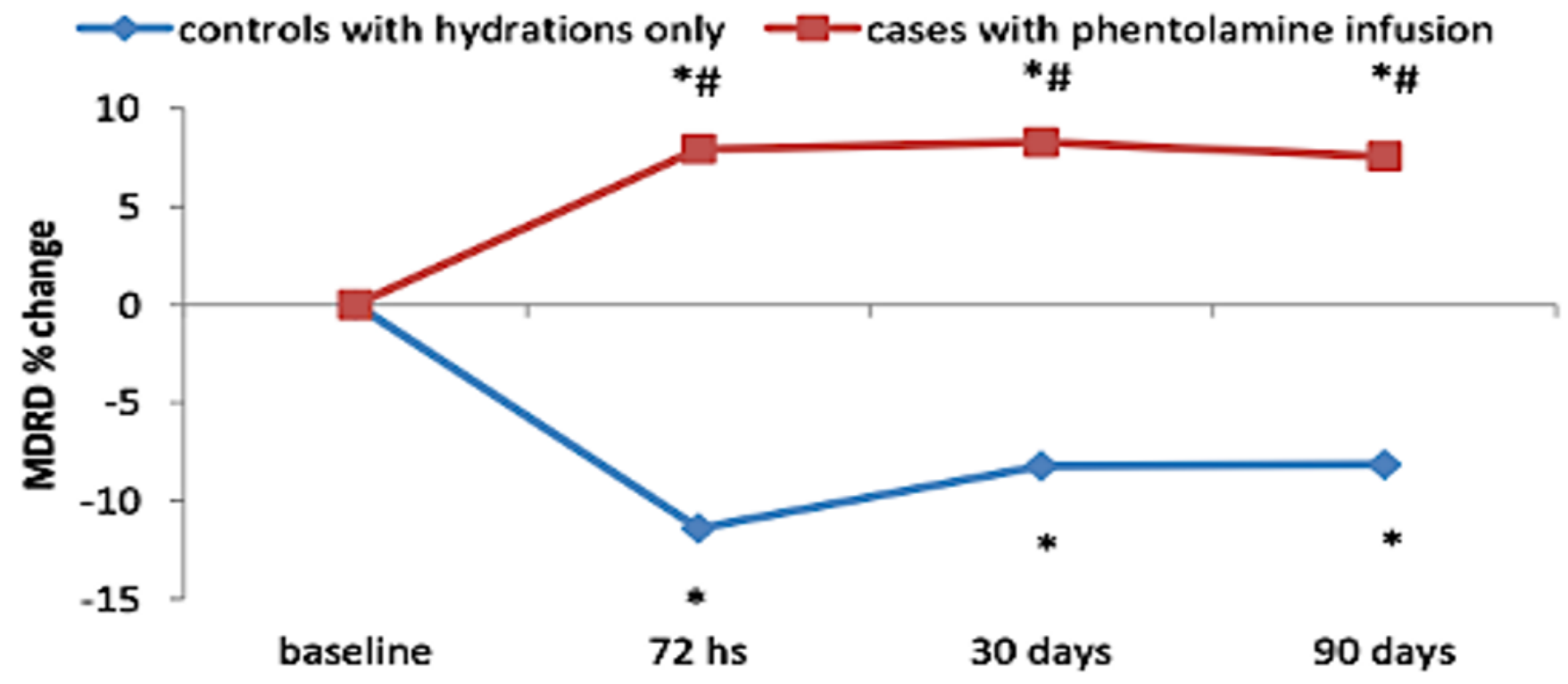

Figure 3 
Change in estimated Glomerular Filtration Rate in group1 versus group 2

Line chart showing the percentage of change in eGFR relative to the baseline eGFR in group 1 at 72 hours post procedure ( $-11.41 \pm 12.33), 30$ days post procedure $(-8.23 \pm 16.90)$, and 90 days post procedure $(-8.16 \pm 17.07)$ versus the percentage of change in eGFR relative to the baseline eGFR in group 2 at 72 hours post procedure $(7.93 \pm 13.05), 30$ days post procedure $(8.28 \pm 20.82)$, and 90 days post procedure (7.55 \pm 24.93$)$ 